\title{
Textile Electrodes for Electrotherapy
}

\author{
Anne Schwarz-Pfeiffer ${ }^{1 *}$ and Lieva Van Langenhove ${ }^{2}$ \\ ${ }^{1}$ Department of Textile and Clothing Technology, Hochschule Niederrhein University of Applied Sciences, Germany \\ ${ }^{2}$ Department of Textiles, Ghent University, Belgium
}

Submission: January 28, 2020; Published: February 14, 2020

*Corresponding author: Anne Schwarz, Department of Textile and Clothing Technology, Hochschule Niederrhein University of Applied Sciences, Webschulstrasse 31, 41065 Moenchengladbach, Germany

\begin{abstract}
Electronics have been present in medicine for decades. Electrocardiography, electromyography and electrotherapy are widely applied methods. While electrodes that are used for monitoring functions are small, the size of electrodes applied in electrotherapy is rather large. The latter electrodes have to be able to supply relatively high currents to the muscles. Due to the muscle contractions caused during the stimulation, skin movement and perspiration may loosen or disrupt the electrode. To accommodate for the relative skin movement under them, the electrodes need to possess sufficient flexibility to ensure good contact to the skin and avoid irritation and skin damage. Against this background textile electrodes integrated into clothes are an attractive alternative against self-adhesive electrodes as they ensure flexibility and a good contact to the human body. In this study, commercially available self-adhesive electrodes are compared to different textile electrodes. The electrodes were placed with a distance of approximately six centimetre on the lower legs of four healthy volunteers. Connected to a commercially available electrostimulation set-up, the different pairs of electrodes are investigated at different current intensities. It was shown that textile electrodes are an attractive alternative towards commercially available electrodes showing a smooth increase in stimulation with increasing current intensities. Keywords: Textile; Electrodes; Electrotherapy; Electrocardiography; Electromyography; Skin damage; Self-adhesive electrodes; Electrical currents; Electroencephalography; Voltage gradients; neutrophils; Macrophages; Physiological changes; Wound healing process
\end{abstract}

\section{Introduction}

Electrotherapy is defined as the treatment of certain human disturbances by applying electrical currents to the body [1]. Endogenous electrical currents which are generated within the body underlie many physiological processes and are recorded during procedures, such as electrocardiography (ECG), electromyography (EMG) and electroencephalography (EEG). Conversely, exogenous electrical currents that are applied to the body from outside are known to produce a variety of effects. One electrotherapy modality is electrical stimulation (ES) [2]. Generally spoken, electrical stimulation is used for three therapeutic purposes: to relieve pain, to stimulate physiological changes and to stimulate muscle contractions (by artificially contract paralyzed muscles with unilateral brain or spinal cord injuries). Above that, electrical stimulation is believed to restart or accelerate the wound healing process by imitating the natural electrical current that occurs in skin when it is injured. In general, pressure wounds are characterized by abnormally low electrical potential, resulting in voltage gradients, compared with the surrounding healthy tissue [3]. Studies on wounded as well as healthy skin tissue showed that electrical stimulation

has positive effects. Applied to wounded tissue electrical current increases the migration of neutrophils and macrophages $[4,5]$ and stimulates fibroblasts [6,7], cells vital to the wound healing process. When applied on healthy tissue, as it is the case in wound prevention, electrical current enhances the blood flow $[8,9]$.

The first electrodes for electrical stimulation on the market (first half of the 20th century) typically consisted of a rubber backing with an indentation into which an absorbent material serving as pad was placed. In the middle of the absorbent pad a metallic electrode plate was mounted [10]. In similar electrode designs, a metal plate in combination with sponges or foams was used [11-13]. In the past 20years bendable and disposable medical electrodes evolved. Instead of a rigid metal plate, a metal mesh, foil or carbon impregnated rubber or vinyl, ensuring more flexibility in the electrode structure, are used. A conductive adhesive layer, usually in form of a gel, is disposed on the conductive side of the material to provide a good electrical conductive contact between the conductive material and the patient's skin. Recently, some producers replaced metal foils 
or meshes with conductive textile materials that provide more flexibility [14]. Aside from the conventional electrode design, textile electrodes in different shapes have been designed. A glove, for instance, made out of silver-coated fabric is presented in [15]. An embroidered textile electrode for electrotherapy is patented in [16]. The above described electrode solutions offer advantages as well as disadvantages. In general, electrodes for electrical stimulation need to provide an electrical signal, distributed over the entire surface of the electrode. Therefore a good, continuous and uniform contact with the skin has to be ensured.

Conventional electrodes placed on the patient's skin by an adhesive conductive surface either tend to loosen due to perspiration and movements during treatment. It may also occur that they stick so well that their removal is painful-another undesired effect. Insufficient flexibility of the electrode can result in severe irritation of the patient's skin and electrical "hot spots" due to uneven electrode-skin contact, manifesting itself in a rash and a burning sensation. The sensation of burning may be sensed by the patient within a few minutes after the application of electrical signals, while the rash conditions generally take longer to develop. Hence, a useful electrode must be drapable in order to accommodate relative movements of the patient's skin. Textile-based electrodes guarantee the desired flexibility. However, the aforementioned examples involve disadvantages as well. The glove is made entirely of a conductive fabric and hence, also the electrode side facing the environment is electroconductive, which can lead to distortions during the treatment. This problem can easily be solved with embroidered electrodes. However, it is very difficult to predict the current density profile in an embroidered electrode, as well as the contact area between the patient's skin and the conductive yarns shaping the electrode. Regarding these considerations it has been decided to exploit different electro-conductive yarns as electrode materials in knitted structures and sewed/laid onto textiles. The following sections concentrate on the prototyping as well as on the electrodes' performance during electrotherapeutic tests on human subjects.

\section{Experimental}

\section{Materials and methods}

\section{Textile electrodes}

Different textile electrode prototypes, varying in yarns and applied production technologies, were designed and developed. For ease of use during testing on human subjects it was decided to focus on the lower leg. Consultation with a medical doctor at the university hospital in Gent showed that the most appropriate placement of the electrodes will be on the back of the calf, in the proximity of the Achilles tendon, as it is fibrous tissue that connects the heel to the muscles of the lower leg, the calf muscles (Figure 1). Different prototypes were designed and developed as described in the following.

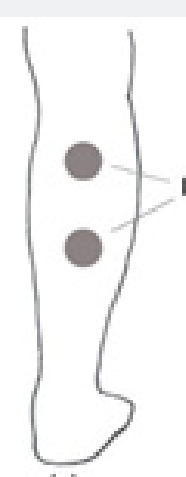

(a)

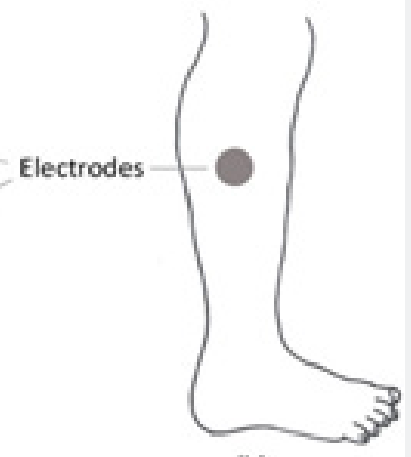

(b)

Figure 1: Positioning electrodes in the proximity of the Achilles tendon:

a) Both electrodes are placed longitudinal along the calf.

b) Electrodes are placed horizontally, aside of the calf.

Stitched and laid electrodes using electro-conductive, elastic yarns

Silver- and copper-based electro-conductive, elastic yarns were integrated into stockings to shape electrodes. The yarns were developed and developed by a Belgian consortium as described in [17]. The electrodes' size was defined to be four centimetres in diameter. Two electrodes per sock were positioned in a distance of six centimetres to each other. The socks used are support stockings to decrease the risk of thrombosis, produced by the Belgian medical textile manufacturer Bota. They were chosen because they guarantee a tight contact with the leg. For stockings with a horizontal arrangement of electrodes aside the calf, the yarns were stitched onto the socks. Connection to the stimulation device was given by a pressure button connection (Figure 2). For the stockings with a longitudinal arrangement of electrodes, the same materials were used as for the stitched electrodes. The electrodes were laid and fix with an upper thread in a zig-zag fashion using an embroidery machine from ZSK, type JCZ 0109-550. These prototypes were produced at the Fraunhofer Institute IZM in Berlin (Figure 3). 


\section{Current Trends in Fashion Technology \& Textile Engineering}

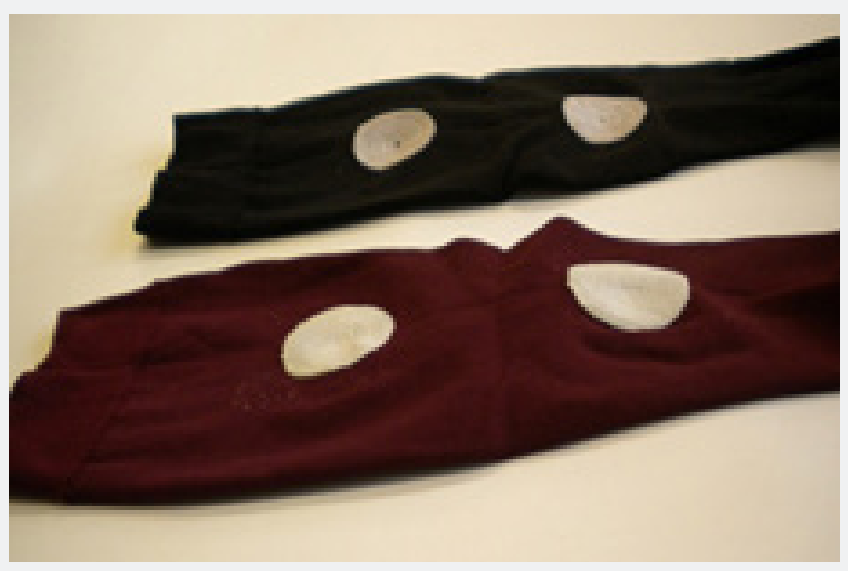

Figure 2: Support stocking prototype with stitched electrodes.
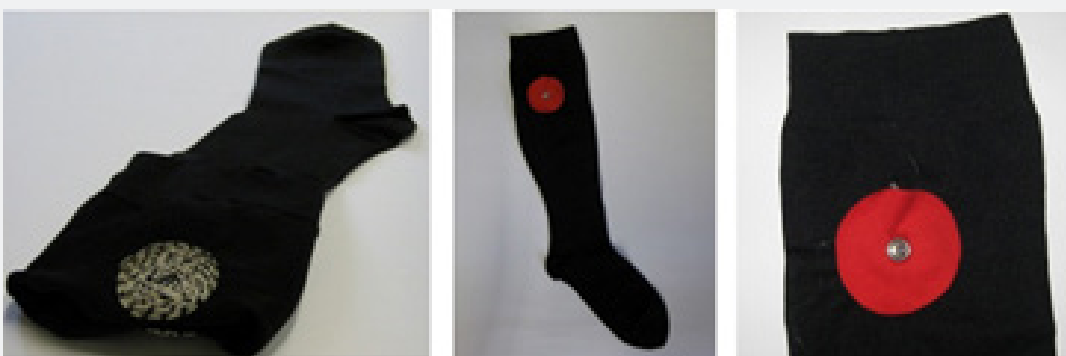

Figure 3: Support stocking prototype with laid (embroidered) electrodes.

Electrodes shaped of gold-coated textiles and sewn onto elastic bands

Gold-coated woven fabrics, produced as described in [18], with the dimension of $4 \times 5 \mathrm{~cm}$ were used for these prototypes. The coated structures were sewn onto elastic bandages, equipped with pressure buttons. Velcro at both ends of the bandages allowed a tight fit around the lower leg (Figure 4).

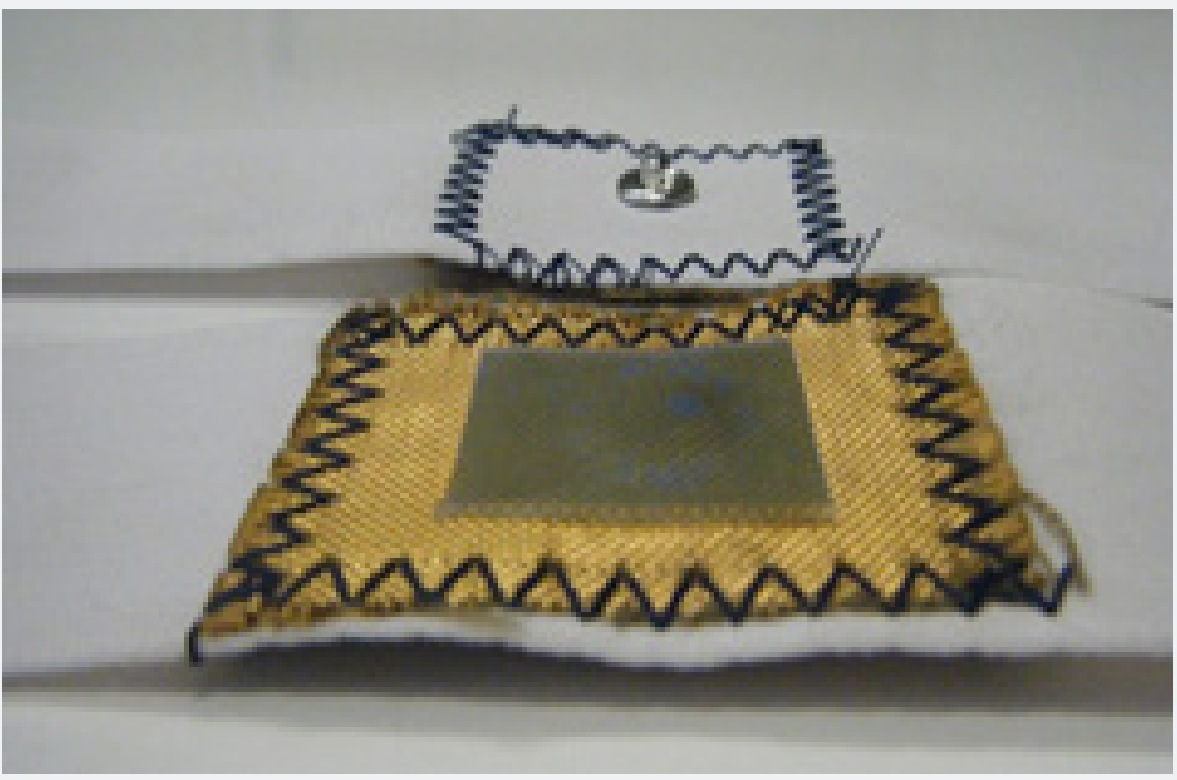

Figure 4: Gold-coated fabric electrodes sewn onto a bandage. 


\section{Knitted electrodes integrated in a knitted bandage}

The knitted bandages with knitted electrodes were produced on a Universal V-double bed flat weft hand knitting machine. The basic structure of the bandage is a rib fabric made of Tactel $®$ yarns. The knitting pattern of the electrode in the bandage uses copper-based electro-conductive, elastic yarn as well as Tactel ${ }^{\circledR}$ yarn. The knitting pattern chosen allows that the electrode is only shaped on one fabric side (Figure 5). The connection of the electrode to the electrotherapeutic device (described in the following section) is established with a pressure button. As in the previously described prototype bandage, Velcro was chosen as fastener.

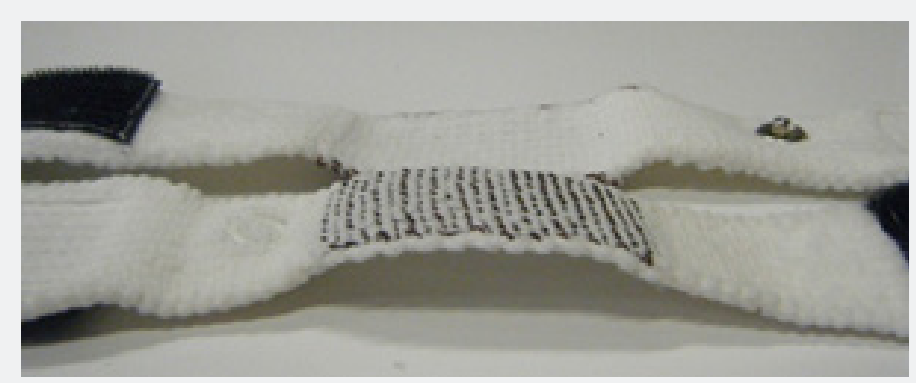

Figure 5: Knitted electrotherapeutic bandages.

\section{Electrostimulation test set-up}

Within the scope of this research, it was not foreseen to include a representative clinical study on the applicability of the textile electrodes for electrotherapy. However, preliminary measurements on the electrodes were performed to give a first indication on their practical use. Hence, the electrodes were tested one four healthy women, not suffering from decubitus, with a portable low-frequency electronic pulse massager, type Omron E4. The device generates voltages up to $90 \mathrm{~V}$ and output currents up to $10 \mathrm{~mA}$, at frequencies reaching $1200 \mathrm{~Hz}$, while being powered with a battery voltage of $3 \mathrm{~V}$ (DC). During the study, different pulses of AC current, with frequencies between $100 \mathrm{~Hz}$ and $1200 \mathrm{~Hz}$ were applied. As a reference, the tests were also run with Omron self-adhesive gel electrodes. The idea was to obtain feedback from the human subjects concerning their experienced sensations during treatment. After the treatment, their skin-arrays, which had contact to the electrode, were examined for possible irritation or damage. Since the placement of the textile electrodes directly on the skin led to frequent turning off of the device, additional hydrogel membranes were employed on textile electrode surfaces as illustrated in (Figure 4). Hydrogel membranes are flexible and can be easily applied on a textile surface, independent of the yarn type.

\section{Results and Discussion}

The results are summarized in (Table 1-7). To allow a comparison between the sensations, they are categorized into four levels, being

Table 1: Preliminary study on volunteers testing different textile electrodes versus conventional electrodes: Stitched electrodes -Silver.

\begin{tabular}{|c|c|c|c|c|}
\hline Level & \multicolumn{4}{|c|}{ Stitched Electrodes - Silver } \\
\hline & Person 1 & Person 2 & Person 3 & Person 4 \\
\hline 1 & - & - & - & Weak \\
\hline 2 & - & Weak & - & Pleasant \\
\hline 4 & - & Weak & Pleasant & Pleasant \\
\hline 6 & - & Pleasant & Pleasant & Pleasant \\
\hline 8 & Weak & Pleasant & Pleasant & Pleasant \\
\hline 10 & Pleasant & Pleasant & Plent & \\
\hline
\end{tabular}

Table 2: Preliminary study on volunteers testing different textile electrodes versus conventional electrodes: Stitched electrodes -Copper.

\begin{tabular}{|c|c|c|c|c|}
\hline Level & \multicolumn{4}{|c|}{ Stitched Electrodes - Copper } \\
\hline & Person 1 & Person 2 & Person 3 & Person 4 \\
\hline 1 & - & Weak & - & Pleak \\
\hline 2 & - & Weak & - & Pleasant \\
\hline 4 & Weak & Pleasant & - & Pleasant \\
\hline 6 & - & Pleasant & - & - \\
\hline
\end{tabular}




\section{Current Trends in Fashion Technology \& Textile Engineering}

\begin{tabular}{|c|c|c|c|}
\hline 8 & Weak & Pleasant & Weak \\
\hline 10 & Painful & Pleasant & Pleasant \\
\hline
\end{tabular}

Table 3: Preliminary study on volunteers testing different textile electrodes versus conventional electrodes: Laid (embroidered) electrodes-Silver.

\begin{tabular}{|c|c|c|c|c|}
\hline Level & \multicolumn{3}{|c|}{ Laid (Embroidered) Electrodes - Silver } \\
\hline & Person 1 & Person 2 & - \\
\hline 1 & - & Person 3 & - \\
\hline 2 & - & Pleasant & - \\
\hline 4 & - & Intensive & Weak \\
\hline 6 & - & Painful & Pleasant \\
\hline 10 & Pleasant & Painful & Pleasant \\
\hline
\end{tabular}

Table 4: Preliminary study on volunteers testing different textile electrodes versus conventional electrodes: Laid (embroidered)electrodes-Copper.

\begin{tabular}{|c|c|c|c|c|}
\hline Level & \multicolumn{3}{|c|}{ Laid (Embroidered) Electrodes - Copper } \\
\hline & Person 1 & Person 2 & Person 3 & - \\
\hline 1 & Weak & - & - \\
\hline 2 & Pleasant & - & - \\
\hline 4 & Pleasant & Weak & - \\
\hline 6 & Pleasant & Weak & Pleasant \\
\hline 8 & Intensive & Pleasant & Pleasant \\
\hline 10 & Intensive & & Pleasant \\
\hline
\end{tabular}

Table 5: Preliminary study on volunteers testing different textile electrodes versus conventional electrodes: Gold-coated textile electrodes.

\begin{tabular}{|c|c|c|c|}
\hline Level & \multicolumn{3}{|c|}{ Gold-Coated Textile Electrodes } \\
\hline & Person 1 & Person 2 & Person 3 \\
\hline 1 & - & - & - \\
\hline 2 & - & Pleasant & Intensive \\
\hline 4 & - & Pleasant & Painful \\
\hline 6 & Intensive & Intensive & Peak \\
\hline 8 & Intensive & Painful & Painful \\
\hline $\mathbf{1 0}$ & Painful & Painful & Painful \\
\hline
\end{tabular}

Table 6: Preliminary study on volunteers testing different textile electrodes versus conventional electrodes: Knitted electrodes.

\begin{tabular}{|c|c|c|c|c|}
\hline \multirow[t]{2}{*}{ Level } & \multicolumn{4}{|c|}{ Knitted Electrodes - Copper } \\
\hline & Person 1 & Person 2 & Person 3 & Person 4 \\
\hline 1 & - & - & Weak & - \\
\hline 2 & - & - & Pleasant & - \\
\hline 4 & - & - & Pleasant & Weak \\
\hline 6 & - & Weak & Pleasant & Pleasant \\
\hline 8 & - & Painful & Painful & Pleasant \\
\hline 10 & - & Painful & Painful & Pleasant \\
\hline
\end{tabular}

Table 7: Preliminary study on volunteers testing different textile electrodes versus conventional electrodes: Conventional OMRON electrodes.

\begin{tabular}{|c|c|c|c|c|}
\hline & \multicolumn{4}{|c|}{ Omron Electrodes } \\
\hline & Person 1 & Person 2 & Person 3 & Person 4 \\
\hline 1 & - & - & Weak & - \\
\hline 2 & - & - & Pleasant & - \\
\hline
\end{tabular}




\section{Current Trends in Fashion Technology \& Textile Engineering}

\begin{tabular}{|c|c|c|c|c|}
\hline 4 & Pleasant & Intensive & Intensive & Pleasant \\
\hline 6 & Intensive & Painful & Painful & Intensive \\
\hline 8 & Painful & Painful & Painful & Painful \\
\hline 10 & Painful & Painful & Painful & Painful \\
\hline
\end{tabular}

a) weak,

b) pleasant/comfortable,

c) intensive/strong stimulation and

d) painful.

First of all, it is noticeable that the pain threshold of each subject is different, which makes it difficult to observe a general trend. However, it can be seen that the usage of the Omron electrodes, as well as the gold textile electrodes and the knitted electrodes caused pain to the human subjects at higher intensities (level 8 and 10). It is well-known that electrostimualtion carries a risk of irritating the skin. During stimulation, a prickling sensation is often felt on the surface of the skin, directly under the electrode. Its cause is not exactly known. According to some sources, it can be attributed to the external stimulation of the nerve endings in the skin $[19,20]$. Others attribute it to thermal effects, which are a result of power dissipation in the skin [21]. Additionally, in some cases, it was noticed that the skin under the electrode turned red. This effect is attributed to vasodilatation of skin capillaries due to electric current flow. Vasodilatation is caused by a release of prostaglandins from skin cells, which is their reaction to the change in their surrounding environment. A subjective feeling of warmth is experienced, which lasts up to several hours after the electrostimulation. As a result of vasodilatation the skin's blood saturation can locally increase by about $500 \%$.

The stitched and the laid (embroidered) electrodes allowed a gentle increase in stimulation intensity. Neither burning sensations nor skin damage were observed after the tests. This indicates that all electrode surfaces are in close and uniform contact with the human skin, which allows a uniform current distribution under the electrode, avoiding the generation of areas with extremely high current concentrations, aggravating damage done to the skin.

\section{Conclusion}

Textile electrodes are an attractive alternative to commercially available self-adhesive electrodes for electrotherapeutic purposes, as they offer flexibility and can be easily integrated into every-day clothing. Different textile electrode prototypes were produced and tested on volunteers against commercial self-adhesive electrodes. When the textile electrodes are equipped with a hydrogel membrane on their surface, they can compete with conventional electrodes in terms of their functionality. However, to fully characterize the textile electrodes for electrostimulation, a controlled clinical study needs to be performed in the near future.

\section{Acknowledgement}

This work has been done in the framework of the EC-cofunded project LIDWINE-Multifunctional Medical Textiles for wound (e.g. Decubitus) prevention and improved wound healing (contract number 026741-2). The financial support of the EC is gratefully acknowledged. Furthermore, the authors would like to express their gratitude to the System on flex group of the Fraunhofer IZM in Berlin for the possibility of embroidering the yarns. Special thanks are also expressed to Philippe Moerkens from Bota to provide socks for this research.

\section{References}

1. Tim Watson, Electrotherapy.

2. Bader D, Bouten C, Colin D, Oomens C (2005) Pressure Ulcer Research: Current and Future Perspectives. Springer 89(2): 1-193.

3. Braddock M, Campbell CJ, Zuder D (1999) Current therapies for wound healing: Electrical stimulation, biological therapeutics, and the potential for gene therapy. International Journal of Dermatology 38(11): 808-817.

4. Eberhardt A, Szczypiorski P, Korytowski G (1982) Effects of transcutaneous electrostimulation on the cell composition of skin exudate. Acta Physiologica Polonica 37(1): 41-46.

5. Orida N, Feldman JD (1982) Directional protrusive pseudopodial activity and mobility in macrophages induced by extracellular electric fields. Cell Motility and the Cytoskeleton 2(3): 243-255.

6. Alvarez OM, Mertz P, Smerbeck R, Eaglstein WH (1983) The healing of superficial skin wound is stimulated by external electrical current Journal of Investigative Dermatology 81(2): 144-148.

7. Cruz NI, Bayron FE, Suarez AJ (1989) Accelerated healing of full thickness burns by the use of high-voltage pulsed galvanic stimulation in the pig. Annals of Plastic Surgery 23(1): 49-55.

8. Hecker B, Carron H, Schwartz DP (1985) Pulsed galvanic stimulation: effects of current frequency and polarity on blood flow in healthy subjects. Arch Phys Med Rehabil 66(6): 369-371.

9. Kaada B (1982) Vasodilation induced by Transcutaneous nerve stimulation in peripheral ischemia. European Heart Journal 3(4): 303314.

10. Frederick H (1926) Surface Electrode For Electrical Therapeutic Apparatus. US Patent 1583087, pp. 1-3.

11. Browner W (1962) Device for Electrical Treatment of Bodily Tissues. US Patent 3055372.

12. Kameny Stanley L (1977) Electrode. US Patent 4014345, pp. 1-4.

13. Donald D Maurer (1974) Electrode for transcutaneous stimulation. US Patent 3817252, pp. 1-4.

14. Axelgaard J, Grussing T (1988) Electrical Simulation Electrode. US Patent 4722354. 
15. Bart Flick A (1994) Electrical Therapeutic Apparatus patent. US 5374283, pp. 1-7.

16. (2006) World Intellectual Property Organization 060934.

17. Schwarz A, Kazani I, Cuny L, Hertleer C, Ghekiere F, et al. (2011) Electro-conductive and elastic hybrid yarns-The effects of stretching cyclic straining and washing on their electro-conductive properties. Materials and Design 32(8-9): 4247-4256.

18. Schwarz A, Hakuzimana J, Kaczynska A, Banaszczyk J, Westbroek P, et al (2010) Gold coated para-aramid yarns through electroless deposition. Surface \& Coatings Technology 204(9-10): 1412-1418.
19. Mason JL, Mackay NA (1976) Pain sensation associated with electrocutaneous stimulation. IEEE Transactions on Biomedical Engineering 23(5): 405-409.

20. Geddes L, Baker LE (1967) The specific resistance of biological material-a compendium of data for the biomedical engineer and physiologist. Medical \& Biomedical Engineering \& Computing 5(3): 271-293.

21. Piekarski K (1967) Some electrical properties of tissue. Electrical Properties of Bone and Cartilage 5: 347-353. 